\title{
Linear reduction of stiffness and vibration frequencies in defected circular monolayer graphene
}

\author{
M. Neek-Amal ${ }^{1}$ and F. M. Peeters ${ }^{2}$ \\ ${ }^{1}$ Department of Physics, Shahid Rajaei Univertsity, Lavizan, Tehran 16788, Iran \\ ${ }^{2}$ Departement Fysica, Universiteit Antwerpen, Groenenborgerlaan 171, B-2020 Antwerpen, Belgium \\ (Received 25 February 2010; revised manuscript received 11 May 2010; published 29 June 2010)
}

\begin{abstract}
Mechanical stiffness of monolayer graphene with randomly distributed vacancies is studied using moleculardynamics simulation and elasticity theory. Nanoindentation is used to obtain Young's modulus and the effective spring constant which decrease linearly with the percentage of vacancies. The load and unload curves are the same and the breaking force and breaking points depend on the percentage of vacancies. Fracture may appear near the boundaries. We introduce a simple method to make the system vibrate by pulling up the atomic force microscopy tip from the center of the clamped circular monolayer graphene which then starts to vibrate.
\end{abstract}

DOI: 10.1103/PhysRevB.81.235437

PACS number(s): $62.25 .-\mathrm{g}$

\section{INTRODUCTION}

Graphene is an almost flat atomic layer of carbon atoms that are densely packed in a honeycomb crystal lattice. ${ }^{1-3}$ The strength of the $s p^{2}$ in plane bonds in graphene provides stability even to a single suspended carbon plane in contrast to crystalline Si or GaAs layers, allowing very light resonators with larger mass or force sensitivity. Those strong bonds cause an extremely high Young's modulus, which together with the large available surface area of these devices enhance the detector's sensitivity as well. Graphene has a Young's modulus as large as diamond and other carbon nanostructures and amounts to about $1 \mathrm{TPa} ., 5$

Some recent studies showed unusual thermomechanical properties of graphene. Anomalous negative thermal expansion and an anomalous temperature dependency of Young's modulus up to $900 \mathrm{~K}$ were predicted by using Monte Carlo simulation for a graphene sample with 8640 atoms. ${ }^{6}$ These unusual behaviors are a consequence of the strong anharmonicity of the graphene sheet. Moreover, Zainalpour-Yazdi and Christofides ${ }^{7}$ showed that both Young's modulus and the binding energy of graphene increases with the area $(A)$ of the graphene nanoribbon as a nonlinear $A^{-1 / 2}$ function for systems smaller than $20 \mathrm{~nm}^{2}$ while there is a linear correlation between these two physical parameters for larger system sizes.

Atomic-scale defects such as vacancies and dislocations change the physical and chemical properties of carbon allotropes. ${ }^{8,9}$ There are experimental techniques available to introduce defects in carbon nanostructures such as electron irradiation, ${ }^{8}$ ion bombardment, ${ }^{10}$ and plasma oxidation. ${ }^{11}$ Transmission electron microscopy and neutron scattering are two common methods to identify the type of defects in graphene. ${ }^{12,13}$

Carlsson and Scheffler ${ }^{14}$ used density-functional theory together with the generalized gradient approximation for the exchange-correlation functional to study electronic and chemical properties of a defected graphene sheet with samples containing 112 atoms in a single supercell. Their study showed that atomic relaxation forms a combination of nonhexagonal rings that leads to local buckling around the defects. There are also several theoretical and experimental studies on defected graphite. ${ }^{15}$ El-Barbary et al. ${ }^{16}$ determined some properties of vacancies in graphite using firstprinciples calculations. They showed that Kohn-Sham eigenvalues near the Fermi level for perfect graphene are lower (around $0.8 \mathrm{eV}$ ) as compared to graphene with a vacancy. In the case of a carbon nanotube Krasheninnikov et al. ${ }^{17} \mathrm{em}$ ployed density-functional theory and found that the formation energy of a double vacancy in single-wall carbon nanotubes is smaller than that for a single vacancy which is a consequence of the nanosize and the unique atomic structure of single-wall carbon nanotubes.

Moreover the actuation and vibration of suspended graphene is another important research subject. There are a few experimental methods to induce vibrations in order to find mechanical resonances such as optical interferometry, ${ }^{4}$ electrostatically actuating and imaging by scanning force microscopy, ${ }^{18}$ nanoindentation of graphene, ${ }^{19}$ and using pressure difference. ${ }^{5}$ The dependence of mechanical vibration frequencies of graphene on the thickness and the size of supported circular graphene have been measured by Poot and van der Zant. ${ }^{20}$ They found an almost linear increase of the frequency versus thickness $(\geq 2 \mathrm{~nm})$ for a sample with $R$ $=540 \mathrm{~nm}$ on the order of $1 \mathrm{GHz}$ and for $R=84 \mathrm{~nm}$ on the order of $10 \mathrm{GHz}$.

In this paper we study the mechanical properties of defected monolayer graphene for three different percentage of vacancies and compare the mechanical response of defected monolayer graphene to the one of perfect monolayer graphene. The Young's modulus of the defected membrane is estimated by using the predictions of the theory of elasticity for a loaded plate in the large deflection regime. Furthermore, the change in bond length of perfect monolayer graphene is investigated. The frequency of vibration is studied by pulling up the center of the clamped membrane using an atomistic atomic force microscopy.

This paper is organized as follows. In Sec. II we will introduce the atomistic model and the simulation method. Section III contains predictions of the theory of elasticity for loaded and vibrating plates. In Sec. IV we give our main numerical results and in Sec. V we will conclude the paper.

\section{MODEL AND METHOD OF CALCULATION}

Classical atomistic molecular-dynamics simulation have been employed to simulate the nanoindentation of a sus- 
pended defected graphene membrane. Initially the coordinates of all atoms in graphene are put in a flat surface of a honeycomb lattice with nearest-neighbor distance equal to $0.142 \mathrm{~nm}$. A rigidly clamped boundary condition was imposed. The shape of the indenters was chosen as a squarebased pyramid in a fcc structure (lattice constant equal to $3.92 \AA$ or $0.392 \mathrm{~nm}$ ) and it is assumed rigid during our simulation. The radius of the circular graphene membrane is $R$ $=15 \mathrm{~nm}$

and three cases of randomly distributed vacancies were considered. The number of atoms in the system is $N_{m}$, the percentage of missing atoms is $p / 100$, which implies $N_{v}=p \times N / 100$ vacancies. In practice we distribute the vacancies in the system, by removing atoms (not boundary atoms) from a perfect sheet with the probability $p / 100$. Algorithmically, for each atom in the prefect sheet, we test this condition: if a uniform random number in the interval $(0,1)$ is less than $p / 100$, the atom is removed. In this study the number of atoms in monolayer graphene are $N_{m}$ $=28.082,27.767,27.496,27.220$ for the systems with $p=0$, $p=1, p=2$, and $p=3$. The number of tip atoms are fixed to $N_{t}=371$ with bottom area $2.02 \mathrm{~nm}^{2}$. We did not allow vacancies at the boundary. We simulated the system at room temperature $300 \mathrm{~K}$ by employing a Nosé-Hoover thermostat. The initial velocities in each direction, were extracted from a Maxwell-Boltzman distribution at the given temperature.

The Brenner's bond-order potential ${ }^{21}$ was used for the carbon-carbon interaction and a Lennard-Jones $(\mathrm{LJ})$ potential $U(r)=4 \varepsilon\left\{(\sigma / r)^{12}-(\sigma / r)^{6}\right\}$ for the indenter-graphene interaction. To model the interaction between the tip and graphene, we use the LJ parameters for Pt atoms with $\varepsilon=68.3 \mathrm{meV}$ and $\sigma=2.54 \AA .^{22}$ For a two-component system, as studied here, the parameters for the mixed interaction between the two types of atoms can be estimated by the simple average suggested by Steel. ${ }^{23}$ In LJ potential, $\sigma$ is the distance at which the potential is zero and $\epsilon$ is the depth of the potential well. Moreover, to save computational time, we truncated the LJ potential at the cut-off distance of $r_{c}=3.5 \sigma$.

After equilibrating the system during 50000 time steps, the lowest position of the tip's atom is located a few angstroms, i.e., $\approx 3.6 \AA$ above the graphene layer. $\Delta t=0.5 \mathrm{fs}$, is the time step in our simulation. The indenter is pushed down slowly with $0.1 \AA$ in a time span of $5000 \Delta t$ which is equivalent to a velocity of $4 \mathrm{~m} / \mathrm{s}$. The indenter atoms interact only with the carbon atoms through the LJ interaction potential. Since we create defects randomly in addition to single vacancies there are a few double vacancies while triplet and higher-order vacancies are very rare in the studied situations.

To vibrate the clamped circular monolayer graphene membrane we pull up the tip slowly with the abovementioned velocity. After the tip is sufficiently retracted (typically $1.75 \mathrm{~ns}$ ) the graphene membrane is released and the membrane starts to vibrate freely without sensing the tip. After this point it has an almost sinusoidal vibration with frequency that depend on $p$ and the size of the monolayer graphene. We sampled a few periods of these vibrations and determined the oscillation frequency.

\section{THEORY OF ELASTICITY}

\section{A. Loaded plate}

The size of the system and the used deflection values ensure that nonlinear elasticity theory ${ }^{24}$ for a circular flake with a large deflection in the $z$ direction is applicable for our graphene circular membrane. In general, the solution of the governing nonlinear differential equation as obtained from elasticity theory for rigidly clamped boundary condition is given by ${ }^{25}$

$$
F=\frac{\pi E h}{4 R^{2}} \frac{1}{G(\nu)} z(r)^{3},
$$

where $r$ is the radial position, $R$ is the radius of the circular plat, $z(r)$ is the deflection at radial position $r, h$ is the thickness of the plate, $E$ is the Young's modulus, and $F$ is the concentrated load on the flake. In Eq. (1) the function $G(\nu)$ is a complicated function of the Poisson ratio. ${ }^{25}$ We use $z(0)$ $=\zeta$ as the graphene deflection at $r=0$. Our computer simulations confirm this behavior for small percentage of vacancies with large deflections of defected graphene which we will discuss in the next section.

The force-displacement curves have been measured recently by Lee et al. $^{26}$ and they showed that it can be approximated by a simple polynomial function having a linear and a cubic term

$$
F=a \zeta+b \zeta^{3} .
$$

When the bending stiffness is negligible and the load is small the force deflection can be approximated by the linear term while the second term dominates for large deflection. ${ }^{27}$ Here we show that for a small percentage of vacancies the same functionality as Eq. (1) for force-deflection holds. The dependence of the parameter $b$ in Eq. (2) on the geometrical and mechanical parameters can be obtained by comparing Eqs. (1) and (2) for the central point which gives the following relation for Young's modulus:

$$
E=\frac{4 b G(\nu) R^{2}}{\pi h},
$$

where $h$ is the thickness and $G$ is a complex function of Poisson's ratio which can be fitted by the line $G(\nu)$ $=-0.593 \nu+0.9365 .^{25}$

\section{B. Free vibrating plate}

Another familiar class of problems in the theory of elasticity is the vibrating shell or plate. ${ }^{24,28}$ The aim of these category of problems is to find the characteristic frequencies of the vibrating plates for different boundary conditions. One of the most important and applicable situation is a clamped circular plate. The equation of motion for free vibration in polar coordinates is

$$
\frac{E h^{3}}{12\left(1-\nu^{2}\right)} \nabla^{4} u(r, \theta, t)+\rho h \frac{\partial u(r, \theta, t)}{\partial t^{2}}=0,
$$

where $\rho$ is the mass density of the plate. For a natural frequency- $\omega$ - we have $u(r, \theta, t)=Z(r, \theta) e^{i \omega t}$ and the timeindependent equation can be written as 


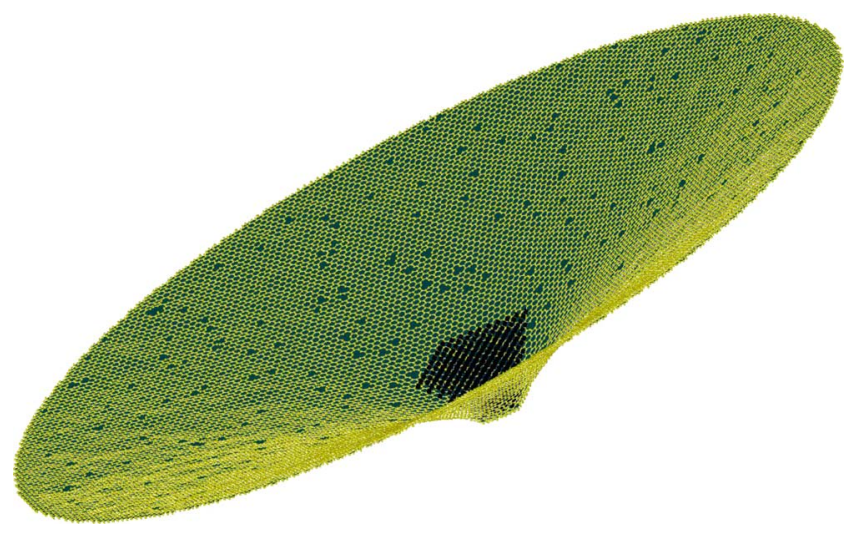

FIG. 1. (Color online) A snap shot of an indenter above circular monolayer graphene with radius $R=15 \mathrm{~nm}$ and $1 \%$ of vacancies. Here we applied clamped boundary condition.

$$
\frac{E h^{3}}{12\left(1-\nu^{2}\right)} \nabla^{4} Z(r, \theta)+\omega^{2} \rho h Z(r, \theta)=0 .
$$

Separating the variables by substituting $Z(r, \theta)=z(r) \Theta(\theta)$ one can solve Eq. (5) exactly for both radial and angular terms. For a closed circular plate the angular term is given by $\cos n(\theta-\phi)$ where $n$ is an integer number. The differential equation for the radial part is Bessel's differential equation. For a perfect plate with clamped boundary condition as $\left.z(r)\right|_{r=R}=0$ and $\left.\frac{\partial z}{\partial r}\right|_{r=R}=0$ the radial solution is a linear combination of the first $\left[J_{n}(r)\right]$ and the second $\left[I_{n}(r)\right]$ kind of Bessel's function, $z(r)=C J_{n}(r)+D I_{n}(r)$. Substituting the solution in the boundary conditions gives an equation for the normal frequencies. Values of the roots of Bessel's function which are counted by the integer $m$ determine the natural frequencies ${ }^{28}$

$$
f_{m n}=\frac{x_{m n}^{2}}{2 \pi R^{2}} \sqrt{\frac{E h^{2}}{12 \rho\left(1-\nu^{2}\right)}},
$$

where $x_{m n}$ are the roots, $x_{00}=3.196, x_{01}=4.611, x_{10}=6.306$, etc.

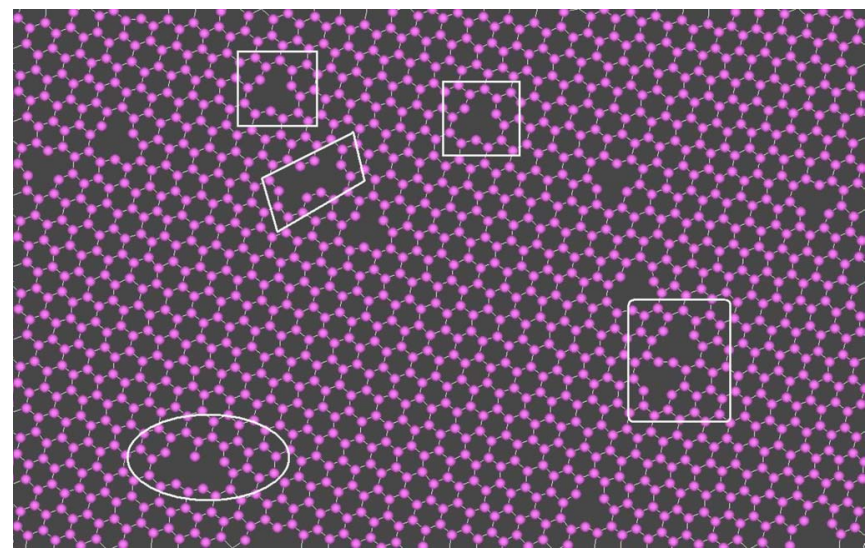

(a)

\section{RESULTS AND DISCUSSION}

Figure 1 shows a snap shot of a circular monolayer graphene membrane with $p=1$ and $R=15 \mathrm{~nm}$. The black atoms are the tip atoms which push the sheet in the center of the membrane. The simulations are done for room temperature. Figure 2 shows two snap shots of part of a graphene sheet, the left-hand side (LHS) picture is the part in the vicinity of the boundary and the right-hand-side picture is the part under the tip, i.e., contact area region. As can be seen from Fig. 2 due to the load, ring structures are found in the contact area. We found that the bond lengths for $p=0$ are larger in the contact area as compared to the other regions.

\section{A. Changing the bond lengths under load}

In the bottom panel of Fig. 3 the radial distribution function, $g\left(R_{n n}\right)$, versus nearest-neighbor distance $R_{n n}$ of the carbon atoms are shown for $p=0$. Notice that loading graphene causes an increase of the mean value of the bond length. The solid curves are two Gaussian fitted functions for the unloaded case with $\zeta=0 \mathrm{~nm}$ and loaded graphene with $\zeta=-3.75 \mathrm{~nm}$ at $T=300 \mathrm{~K}$. The mean values of the Gaussian function are $R_{m}=1.426 \AA$ and $R_{m}=1.439 \AA$, and standard deviations are $0.0332 \AA$ and $0.0352 \AA$, respectively. The variation of $R_{m}$ with respect to the deflection values are shown in the top panel of Fig. 3. Dashed line is the best fitted curve to the data for $R_{m}$. This curve is quadratic with respect to the deflection value: $R_{m}=1.426125+0.00102 \zeta^{2}$. This behavior is different from the dependence of $R_{m}$ and its variance with respect to temperature where $R_{m}$ is unaltered due to temperature while the variance increases as $\sqrt{T}$ below $T=900$ K. $^{6}$

\section{B. Mechanical stiffness}

The $z$ component of the forces applied to the graphene atoms from the indenter are calculated by summing over the total reaction forces

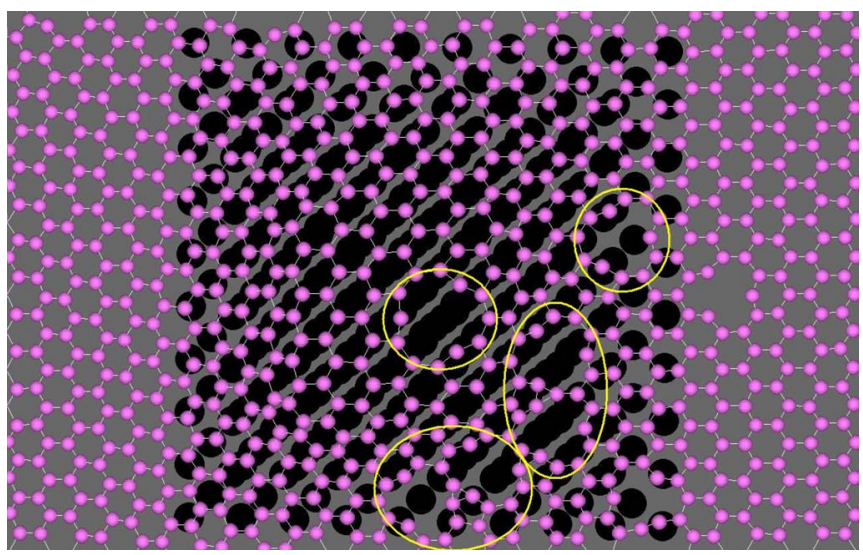

(b)

FIG. 2. (Color online) Two enlarged parts of Fig. 1. Left: some typical defects in the graphene sheet with $1 \%$ vacancies. Right: rings of $\mathrm{C}$ atoms appear around vacancies in the tip contact area (black square region). This snap shot is taken close to the breaking threshold. 

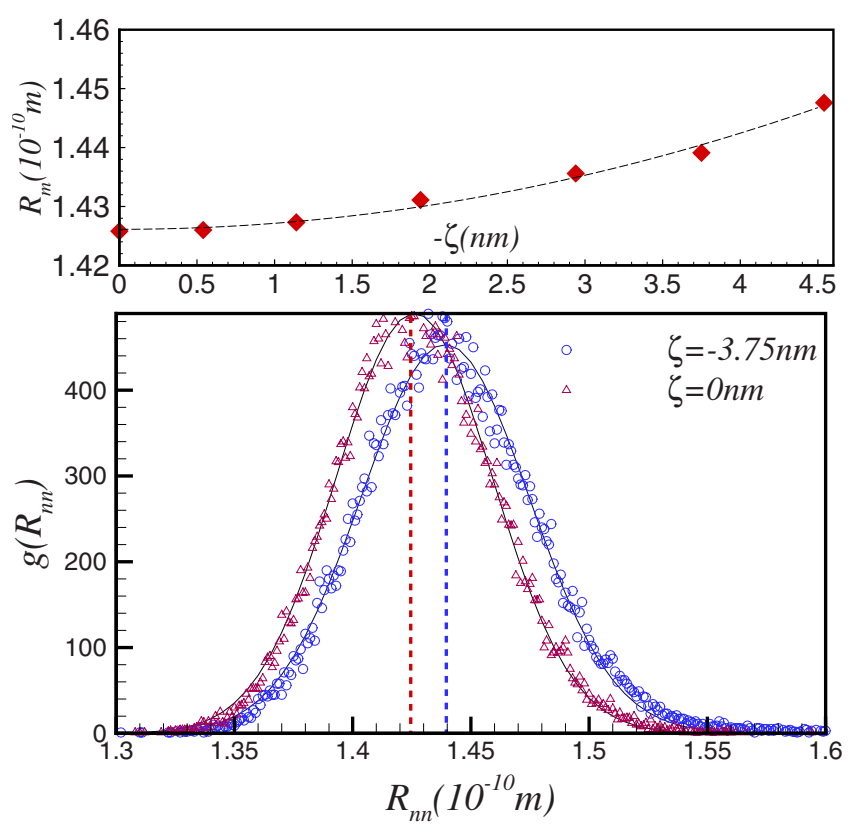

FIG. 3. (Color online) Bottom: radial distribution function of the first nearest-neighbor distance, $R_{n n}$, for unloaded monolayer graphene and loaded graphene at $\zeta=-3.75 \mathrm{~nm}$ for $p=0$. The solid lines show two Gaussian fits. Dashed vertical lines indicate the mean value of the Gaussian fits. Top: the variation of the mean value of the Gaussian fits versus deflection. The dashed curve is a quadratic fit to the simulation data.

$$
F=\sum_{i=1}^{N_{t}} \sum_{j=1}^{N_{b}} F_{i j}^{z}=24 \varepsilon \sum_{k=1}^{2}\left\{\sum_{i=1}^{N_{t}} \sum_{j=1}^{N_{b}}(-1)^{k} 2^{k-1}\left(\frac{\sigma}{r_{i j}}\right)^{6 k} \frac{z_{i j}}{r_{i j}^{2}}\right\} .
$$

Because of the short-range nature of the potentials, the sum over $N_{t}$ and $N_{m}$ can be truncated and limited to a few neighbors. The first term, $k=1$ in the parentheses, is the derivative of the attractive part of the LJ potential and the second term $(k=2)$ is related to the derivative of the repulsive term.

Figure 4 shows the variation of the applied load at $r=0$ as a function of the deflection in the $z$ direction. The plotted force was obtained as follows. After each 5000 time steps the tip was pushed down with $0.1 \AA$ in order to induce the deflection $\zeta$. During this time interval we let the system equilibrate and in the last 1000 time steps of these intervals we calculated $F$ and obtained the mean value of $F$. Thus in a simulation with $10^{6}$ time steps we have 200 points in Fig. 4.

As can be seen from Fig. 4 increasing the number of vacancies reduces the force on the tip for fix displacement value as is expected because, the number of interacting atoms on the tip decreases with increasing $p$. We fitted a function $F=a \zeta+b \zeta^{3}$ to the results, according to Eq. (2), which are shown as solid curves in Fig. 4. Here $\zeta$ is the deflection value in the central point.

In Fig. 5 the variation of the two parameters versus the defects percentage are shown. Notice that $a$ has almost a linear dependence that can be fitted to $a=-0.84 p+3.085$ and $b=-0.388 p+7.09$. Decreasing $a$ gives a weaker effective spring constant for monolayer graphene. Applying the theory

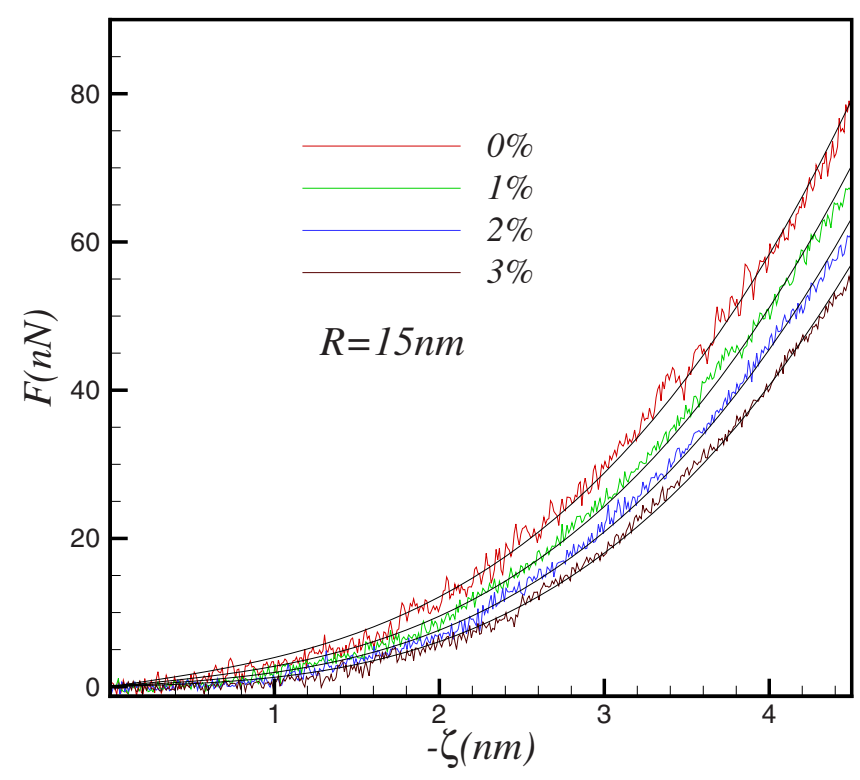

FIG. 4. (Color online) The force deflection data for monolayer graphene with $p \%$ defects (vacancy) in the system. The radius of the membrane is $R=15 \mathrm{~nm}$ and temperature is $T=300 \mathrm{~K}$. The defects were randomly distributed in the graphene sheet. Solid curves are fitted results using Eq. (2).

of elasticity for large deflection, ${ }^{25}$ one can find the Young's modulus according to Eq. (3). Using Eq. (3) and the almost linear function $G(\nu)$ obtained in Ref. $25[G(\nu \approx 0.25)$ $=0.83]$ with $h=3.45 \AA$ our simulation results for Young's modulus are listed in Table I. These numbers are comparable to those found in nanoindentation experiments, i.e., $1.0 \pm 0.1 \mathrm{TPa}$ in Ref. 26 and $0.5 \pm 0.1 \mathrm{TPa}$ in Ref. 19. Arroyo and Belytschko ${ }^{29}$ calculated $E$ equal to $0.7 \mathrm{TPa}$ by using Tersoff-Brenner force field. Note that employing smaller val-
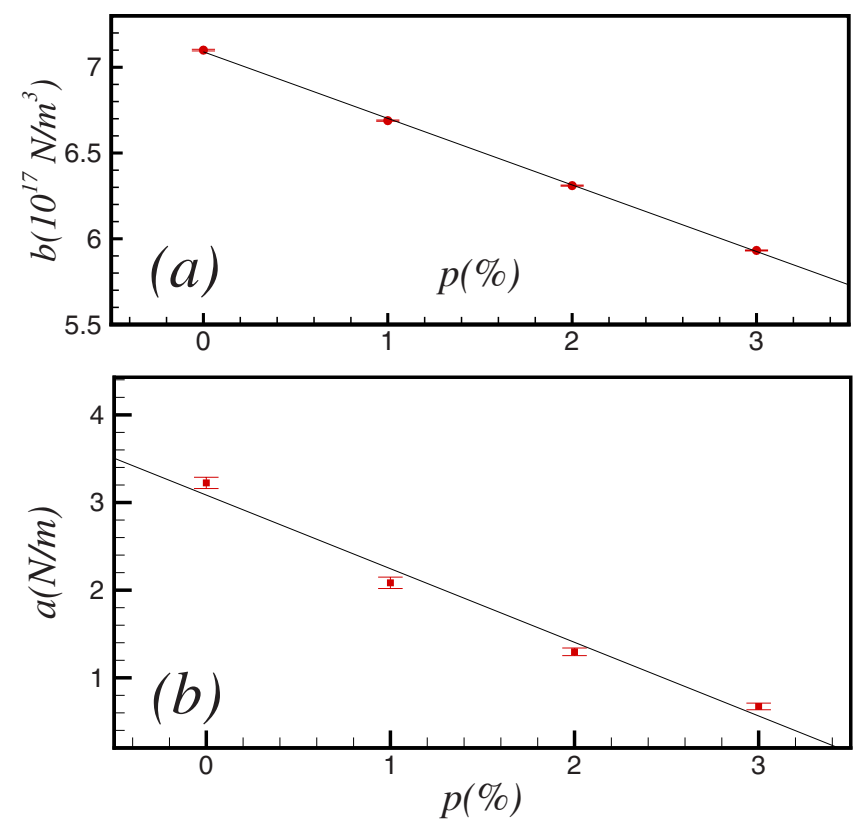

FIG. 5. (Color online) Variation of the coefficient (a) $b$ and (b) $a$ in Eq. (2) versus $p$. The solid lines are linear fits. Error bars are of the order 0.005 in (a) and on the order 0.05 in (b). 
TABLE I. Young's modulus calculated by using Eq. (3). The calculated frequencies using moleculardynamics simulation, $f_{m d}$. Prediction from elasticity theory for the frequencies $f_{t}$ using Eq. (8).

\begin{tabular}{cccccc}
\hline \hline & $\begin{array}{c}E \\
(\mathrm{TPa})\end{array}$ & $\begin{array}{c}f_{m d} \\
(\mathrm{GHz})\end{array}$ & $\begin{array}{c}f_{t} \\
(\mathrm{GHz})\end{array}$ & $\begin{array}{c}F_{b} \\
(\mathrm{nN})\end{array}$ & $\begin{array}{c}\zeta_{b} \\
(\mathrm{~nm})\end{array}$ \\
\hline 0.0 & $0.501 \pm 0.032$ & $20.0 \pm 1.5$ & 17.2 & $80 \pm 5$ & $5.0 \pm 0.3$ \\
1.0 & $0.471 \pm 0.025$ & $16.0 \pm 1.0$ & 16.8 & $70 \pm 4$ & $4.8 \pm 0.3$ \\
2.0 & $0.442 \pm 0.022$ & $8.9 \pm 0.9$ & 16.3 & $65 \pm 4$ & $4.5 \pm 0.4$ \\
3.0 & $0.413 \pm 0.019$ & $4.0 \pm 0.5$ & 15.8 & $55 \pm 4$ & $4.4 \pm 0.4$ \\
\hline \hline
\end{tabular}

ues for the thickness of monolayer graphene ${ }^{2}$ causes an increase in the Young's modulus. If we take the thickness equal to the carbon-carbon bond length, i.e., $h \simeq 1.42 \AA$ the Young's modulus increases to $E=1.2 \mathrm{TPa}$ for $p=0$. The linear reduction in the Young's modulus with defect concentration can be used experimentally to infer the defect concentration in graphene.

In the case of perfect graphene the fractured area of the membrane occurs under the tip, i.e., in the contact area, see Fig. 2. But for defected graphene when $p \neq 0$ it may occur in other points even in the vicinity of the boundary. Usually the breaking threshold occurs for deflections around $5 \mathrm{~nm}$ for $p=0$ which decreases with increasing $p$. The breaking forces $\left(F_{b}\right)$ and the breaking deflections $\left(\zeta_{b}\right)$ are listed in Table I. In the experiments of Ref. 26 on perfect graphene, they employed two micron-size circular samples with radii 500 and $750 \mathrm{~nm}$, and two thick indenter with radii 16.5 and $27.5 \mathrm{~nm}$. The obtained breaking forces were $1770 \mathrm{nN}$ and $2980 \mathrm{nN}$ for tips with radius $16.5 \mathrm{~nm}$ and $27.5 \mathrm{~nm}$, respectively. The ratio between the breaking deflection and the sample radius, varies from 0.2 to 0.3 for tip size 16.5 to $27.5 \mathrm{~nm}$. We found for this ratio $\zeta_{b} / R \cong 0.33$ which is not much different from the experimental values. In our study we used a small tip (with an effective radius, in the order of $1 \mathrm{~nm}$ ) above a circular sample. Since the bond lengths increases at the surface boundary and the probability of finding two double or higher order vacancies in the vicinity of each other is considerable (see LHS of Fig. 2, the out of the center region) and this probability increases with $p$ hence the fracture region might appear out of the center for $p \neq 0$. Furthermore, the breaking force is reduced when increasing $p$. Therefore the fracture area position depends on $p$. According to our simulation results the same load and unload force deflection curves are found independent of the vacancy percentage.

\section{Free vibrating frequencies}

For actuating monolayer graphene we pull up the tip and after separating the tip from monolayer graphene which occurs when the total force on the tip become zero, we extracted a series of $z$ component of monolayer graphene versus time (for an $\sim$ ns range). Figure 6 shows the variation of the $z$ positions of the center of mass of monolayer graphene for the cases $p=0$ and $p=3 \%$. Calculating the period of the vibration, i.e., the frequency $f_{m d}$ which is listed in Table I. As can be seen the frequency decreases with increasing the percentage of vacancies. This is because of the reduction of stiffness of graphene which is a consequence of the reduced mass density and missed $s p^{2}$ bonds in the sheet. Results are in good agreement with experimental results where the vibrating frequencies are in the gigahertz range. ${ }^{20}$ The frequency of the fundamental mode for two graphene samples decreases versus the radius [it is in the $[0.2-1.0] \mathrm{GHz}$ range for $R=540 \mathrm{~nm}$ and is in the [5.0-11.0] GHz range for $R$ $=84 \mathrm{~nm}$ (Ref. 20)]. Therefore, it is expected that for smaller system, $R=15 \mathrm{~nm}$ in our simulation, we obtain higher frequencies (Table I). We can also compare our results with the prediction from elasticity theory by using Eq. (6). For monolayer graphene one needs values for the mass density and the Poisson's ratio. Assuming $\rho=2200\left(\mathrm{Kg} / \mathrm{m}^{3}\right)$ (Ref. 18) and $\nu \sim 0.165$ (Ref. 4) for perfect circular graphene with $R$ $=15 \mathrm{~nm}$ and a simple guess for the defected case which we look as $\rho(p)=\rho(1-p / 100)$ we find for the frequencies

$$
f_{t}=\frac{x_{m n}^{2}}{2 \pi R} \sqrt{\frac{b G h}{3 \pi \rho\left(1-\nu^{2}\right)}},
$$

where typically we have $x_{m n} \sim 4$. Results are listed in the last column of Table I. Another estimate of the frequency for perfect circular graphene can be made by using the mass density $\rho=0.76\left(\mu \mathrm{g} / \mathrm{m}^{2}\right)$ (Ref. 2) and $\nu \sim 0.165,{ }^{4}$ which gives $f_{t}=16.4 \mathrm{GHz}$.

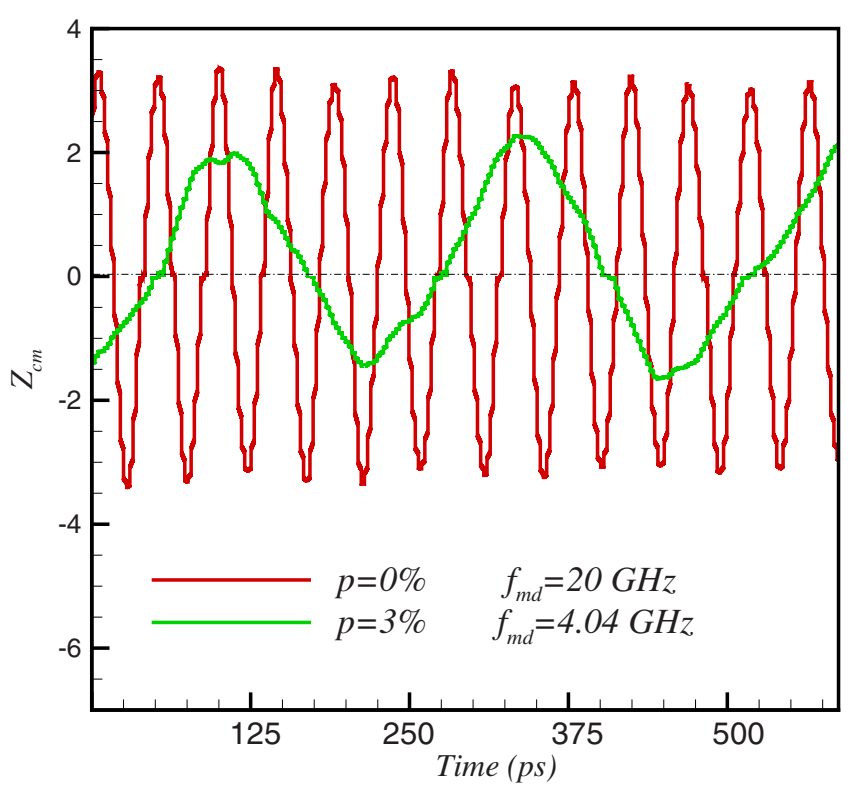

FIG. 6. (Color online) Variation of the $z$ positions of the center of mass of graphene versus time for two cases $p=0 \%$ and $3 \%$. 
Comparing the results for $f_{m}$ and $f_{t}$ shows that the above assumption for the mass density of defected graphene is reasonable for small percentage of vacancies and one needs a more advanced theory for the reduction in mass density and Poisson's ratio to explain defected graphene. The theory of elasticity predicts a much smaller decrease of the frequency as compared to our simulations which is due to nonlinear effects.

Furthermore, we did two extra simulations at $T=20 \mathrm{~K}$ for $p=0 \%$ and $p=3 \%$. The Young's modulus decreases by $5 \%$ and $13 \%$ for $p=0 \%$ and $p=3 \%$, respectively, with respect to the $T=300 \mathrm{~K}$ case, see Ref. 6 . The vibration frequency for the system at $20 \mathrm{~K}$ with $p=0 \%$ is almost the same as for $T$ $=300 \mathrm{~K}$ while for $p=3 \%$ it decreases by a factor of 5 .

\section{CONCLUSION}

In this study we showed that defected monolayer graphene has a lower stiffness with respect to perfect mono- layer graphene. Young's modulus for defected graphene was found to linearly decrease versus the percentage of vacancies. The force-displacement result could be fitted to the function $F=a \zeta+b \zeta^{3}$. We found that for given displacement the exerted force on defected graphene is smaller than the one for perfect graphene. We introduced a simple method to vibrate the graphene sheet and showed that the frequency of vibration obtained from our molecular-dynamics simulation is in good agreement with experimental results. ${ }^{20} \mathrm{We}$ compared our results to those obtained from elasticity theory and found that only for small percentage of vacancies, the predictions of elasticity theory, and our simulation results are close while for larger density they deviate due to nonlinear effects.

\section{ACKNOWLEDGMENT}

This work was supported by the Flemish Science Foundation (FWO-Vl) and the Belgium Science Policy (IAP).
${ }^{1}$ A. K. Geim and K. S. Novoselov, Nature Mater. 6, 183 (2007); A. H. Castro Neto, F. Guinea, N. M. R. Peres, K. S. Novoselov, and A. K. Geim, Rev. Mod. Phys. 81, 109 (2009).

${ }^{2}$ T. J. Booth, P. Blake, R. R. Nair, D. Jiang, E. W. Hill, U. Bangert, A. Bleloch, M. I. Gass, K. S. Novoselov, M. I. Katsnelson, and A. K. Geim, Nano Lett. 8, 2442 (2008).

${ }^{3}$ K. S. Novoselov, A. K. Geim, S. V. Morozov, D. Jiang, Y. Zhang, S. V. Dubonos, I. V. Grigorieva, and A. A. Firsov, Science 306, 666 (2004).

${ }^{4}$ J. S. Bunch, A. M. van der Zande, S. S. Verbridge, I. W. Frank, D. M. Tanenbaum, J. M. Parpia, H. G. Craighead, and P. L. McEuen, Science 315, 490 (2007).

${ }^{5}$ J. S. Bunch, S. S. Verbridge, J. S. Alden, A. M. van der Zande, J. M. Parpia, H. G. Craighead, and P. L. McEuen, Nano Lett. 8, 2458 (2008)

${ }^{6}$ K. V. Zakharchenko, M. I. Katsnelson, and A. Fasolino, Phys. Rev. Lett. 102, 046808 (2009).

${ }^{7}$ C. D. Zeinalipour-Yazdi and C. Christofides, J. Appl. Phys. 106, 054318 (2009).

${ }^{8}$ A. Hashimoto, K. Suenaga, A. Gloter, K. Urita, and S. Iijima, Nature (London) 430, 870 (2004).

${ }^{9}$ K. Suenaga, H. Wakabayashi1, M. Koshino, Y. Sato, K. Urita, and S. Iijima, Nat. Nanotechnol. 2, 358 (2007).

${ }^{10}$ J. R. Hahn, H. Kang, S. Song, and I. C. Jeon, Phys. Rev. B 53, R1725 (1996).

${ }^{11}$ J. I. Paredes, A. Martinez-Alonso, and J. M. D. Tasco'n, Carbon 38, 1183 (2000).

${ }^{12}$ Jannik C. Meyer, C. Kisielowski, R. Erni, Marta D. Rossell, M. F. Crommie, and A. Zettl, Nano Lett. 8, 3582 (2008).

${ }^{13}$ M. H. Gass, U. Bangert, A. L. Bleloch, P. Wang, Rahul R. Nair, and A. K. Geim, Nat. Nanotechnol. 3, 676 (2008).

${ }^{14}$ J. M. Carlsson and M. Scheffler, Phys. Rev. Lett. 96, 046806 (2006).
${ }^{15}$ J. G. Kushmerick, K. F. Kelly, H. P. Rust, N. J. Halas, and P. S. Weiss, J. Phys. Chem. B 103, 1619 (1999); K. F. Kelly, D. Sarkar, G. D. Hale, S. J. Oldenburg, and N. J. Halas, Science 273, 1371 (1996); Z. Tang, M. Hasegawa, T. Shimamura, Y. Nagai, T. Chiba, Y. Kawazoe, M. Takenaka, E. Kuramoto, and T. Iwata, Phys. Rev. Lett. 82, 2532 (1999); P. A. Thrower, Chem. Phys. Carbon 5, 217 (1969).

${ }^{16}$ A. A. El-Barbary, R. H. Telling, C. P. Ewels, M. I. Heggie, and P. R. Briddon, Phys. Rev. B 68, 144107 (2003).

${ }^{17}$ A. V. Krasheninnikov, P. O. Lehtinena, A. S. Fostera, and R. M. Nieminena, Chem. Phys. Lett. 418, 132 (2006).

${ }^{18}$ D. Garcia-Sanchez, A. M. van der Zande, A. San Paulo, B. Lassagne, P. L. McEuen, and A. Bachtold, Nano Lett. 8, 1399 (2008).

${ }^{19}$ I. W. Frank, D. M. Tanenbaum, A. M. van der Zander, and P. L. McEuen, J. Vac. Sci. Technol. B 25, 2558 (2007).

${ }^{20}$ M. Poot and H. S. J. van der Zant, Appl. Phys. Lett. 92, 063111 (2008).

${ }^{21}$ D. W. Brenner, Phys. Rev. B 42, 9458 (1990).

${ }^{22}$ Ş. Erkoç, in Annual Reviews of Computational Physics IX, edited by D. Stauffer (World Scientific, 2001), pp. 1-103.

${ }^{23}$ H. A. Steel, The Introduction of Gases with Solid Surfaces (Pergamon, Oxford, 1974).

${ }^{24}$ L. D. Landau, L. P. Pitaevskii, A. M. Kosevich, and E. M. Lifshitz, Theory of Elasticity (Pergamon, Oxford, 1986).

${ }^{25}$ C. Jin, Appl. Math. Mech., Engl. Educ. 29, 889 (2008).

${ }^{26}$ C. Lee, X. Wei, J. W. Kysar, and J. Hone, Science 321, 385 (2008).

${ }^{27}$ S. Timoshenko and S. W. Krieger, Theory of Plates and Shells (McGraw Hill, New York, 1984).

${ }^{28}$ W. Soedel, Vibration of Shells and Plates (Marcel Dekker, New York, 1993).

${ }^{29}$ M. Arroyo and T. Belytschko, Phys. Rev. B 69, 115415 (2004). 